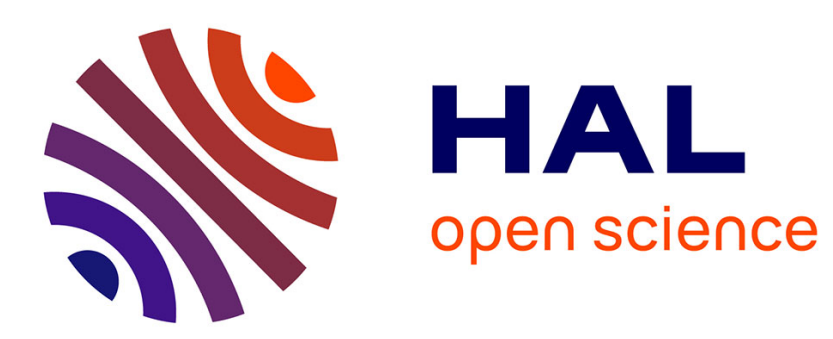

\title{
Poppy Humanoid Platform: Experimental Evaluation of the Role of a Bio-inspired Thigh Shape
}

Matthieu Lapeyre, Pierre Rouanet, Pierre-Yves Oudeyer

\section{To cite this version:}

Matthieu Lapeyre, Pierre Rouanet, Pierre-Yves Oudeyer. Poppy Humanoid Platform: Experimental Evaluation of the Role of a Bio-inspired Thigh Shape. Humanoids 2013, Oct 2013, Atlanta, United States. hal-00861110

\author{
HAL Id: hal-00861110 \\ https://hal.inria.fr/hal-00861110
}

Submitted on 11 Sep 2013

HAL is a multi-disciplinary open access archive for the deposit and dissemination of scientific research documents, whether they are published or not. The documents may come from teaching and research institutions in France or abroad, or from public or private research centers.
L'archive ouverte pluridisciplinaire HAL, est destinée au dépôt et à la diffusion de documents scientifiques de niveau recherche, publiés ou non, émanant des établissements d'enseignement et de recherche français ou étrangers, des laboratoires publics ou privés. 


\title{
Poppy Humanoid Platform: Experimental Evaluation of the Role of a Bio-inspired Thigh Shape
}

\author{
Matthieu Lapeyre ${ }^{1}$, Pierre Rouanet ${ }^{1}$ and Pierre-Yves Oudeyer ${ }^{1}$
}

\begin{abstract}
In this paper, we present an experimental evaluation of the role of the morphology in the Poppy humanoid platform. More precisely, we have investigated the impact of the bio-inspired thigh, bended of $6^{\circ}$, on the balance and biped locomotion. We compare this design with a more traditional straight thigh. We describe both the theoretical model and real experiments showing that the bio-inspired thigh allows the reduction of falling speed by almost $60 \%$ (single support phase) and the decrease of the lateral motion needed for the mass transfer from one foot to the other by $30 \%$ (double support phase). We also present an experiment where the robot walks on a treadmill thanks to the social and physical guidance of expert users and we show that the bended thigh reduces the upper body motion by about $45 \%$ indicating a more stable walk.
\end{abstract}

\section{INTRODUCTION}

Humanoid robots need to be able to move robustly and efficiently in human environments, which includes the ability to keep stability when unpredictable physical contact with humans happens. This raises new important challenges regarding the robustness and safety of robots in an always changing, unpredictable, and open-ended environment.

While state-of-art humanoid and bipedal walking robots (e.g. Honda Asimo [1] or Kawada Industries HRP robots [2]) have shown interesting results on the achievement of efficient walking behaviors in a rather specified environment, they did not really explore robustness capabilities in unspecified environment and reactivity under unpredicted events. Yet, one will have to tackle these challenges to be safely used at home with non-expert users. Also, their walking behaviors require high speed sensorimotor control loop using inverse dynamic and zero moment point [3] with precise and powerful actuation. We think that another interesting way to enable robots to adapt their behaviors to unknown environments is to provide them with control algorithms which can be updated with learning algorithms based either on social guidance [4], or on autonomous self-exploration [5]. A part of the computation needed for such adaptation could also be done through the intrinsic mechanics and electronics of the robot, thus providing effective and hyper-responsive reactions while simplifying the algorithms of the different behaviors. This role of morphology has been called morphological computation [6], as the body of the robot becomes a form of information processing structure [7], able to support a part of the computation necessary to achieve

*This research was partially funded by ERC Starting Grant EXPLORER 240007.

${ }^{1}$ INRIA Flowers Team, Bordeaux, France matthieu. lapeyre, pierre.rouanet, pierre-yves.oudeyer at

inria.fr

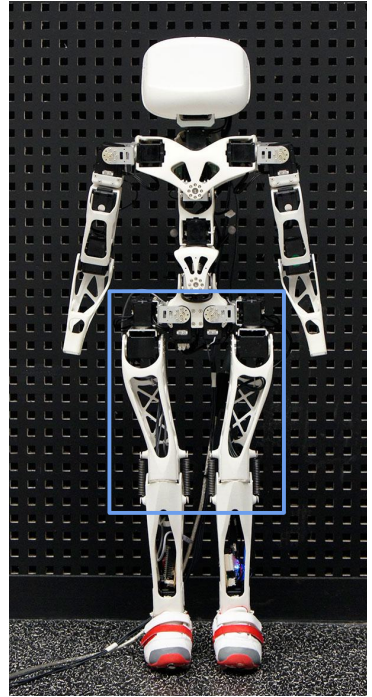

(a) bended thighs

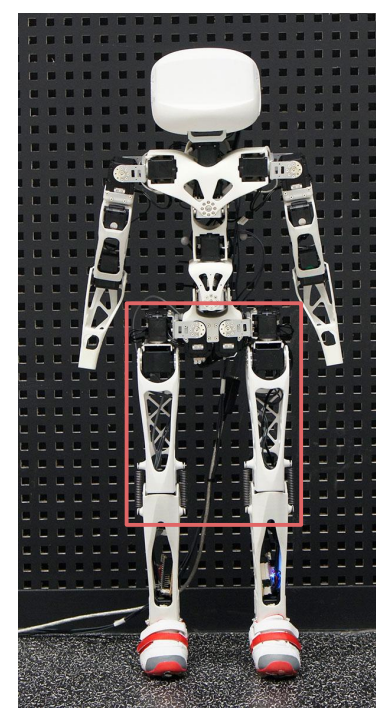

(b) straight thighs
Fig. 1: We evaluate the effect of the thigh morphology on the biped locomotion dynamic. Experiments are made using the Poppy humanoid platform. In this paper, we compare two thigh morphologies: (a) thigh bended by an angle of $6^{\circ}$ and (b) a more classical approach with straight thighs.

sensorimotor tasks to simplify or make it more robust to external disturbances [8]. The actions or reactions of the physical body also have the advantage of being direct without latency due to a controller, as opposed to CPU computed reactions which often require high-cost hardware in order to respond fast enough and reduce modeling errors.

Within this context we built a whole new humanoid robot called Poppy (see Fig. 1a) presented with details in [9]. This humanoid robot was designed to easily and quickly conduct scientific experiments on sensorimotor learning, exploring morphology properties, and human-robot interaction. As an experimentation robotic platform, Poppy was designed to be affordable, lightweight, robust and safe, easy to use, versatile and fast and easy to duplicate or modify with the mid-term goal to make it easily reproducible by other lab through an OpenSource distribution (hardware and software). This was achieved thanks to 3D printing techniques, affordable off-the-shell components and optimized mounting design.

Its morphology is optimized on the locomotive system (legs and trunks) to increase the robot robustness, agility and stability during the walking. This is ensured by a 
combination of an anthropomorphic morphology, morphological computation, articulated spine and lightweight/soft material. Poppy's morphological properties are theoretically described in [9]. In this paper we will focus on its bioinspired thigh shape, bended by an angle of $6^{\circ}$. We will investigate the impact of this thigh design on the balance and biped locomotion using a comparison with a more traditional straight thigh (see Fig. 1).

In the rest of the paper, we will first review current studies on the role of the morphology for biped locomotion. Then we will present an overview of morphological properties and detail the design of the thigh morphology. Afterwards we will present experiments we did to compare the current thigh shape (see Fig. 1a) with a more classical approach to humanoid leg morphology (see Fig. 1b) and finally present and discuss results showing the impacts of its morphology on biped locomotion dynamic.

\section{RELATED WORK}

Most of the studies made on the humanoid robot locomotion in the past 30 years [10] [11] [10] mainly focus on tackling the challenge of biped walking through the active control of the whole robot dynamics using technics such as ZMP control [12] requiring very precise and high torque actuation [13].

The properties of the robot morphology have shown interesting results for robust locomotion, for instance the hexapod robot Rhex [14]. Still, it is surprising that only few explored the challenge of biped locomotion through the study of the role of morphology. One can cite the work of Chandana Paul and Josh C.Bongard [15] and Ken Endo [16] which have explored evolutionary optimization on robot morphology to achieve stable biped locomotion. They have showed a strong impact of the morphology on the walking behavior and were able to reduce the complexity of the controller by finding good mechanical properties (limbs length and mass distribution). It has also been shown that human morphological properties such as the compliance of the body explains the dynamics of walking and running [17] while experiments made by Kojiro Matsushita [18] show that an adequate morphology is needed if one is interested in natural looking kind of locomotion.

The role of morphology in robot biped locomotion has been particularly explored through the research on passive dynamic walkers [19]. The most famous example concerns the Tad MacGeer's work [20]. Thanks to the understanding of the intrinsic dynamics of its structure, McGeer has managed to create a $2 \mathrm{D}$ biped robot capable of producing several steps without any controller or motor. The only control of this robot is obtained through the interaction between the intrinsic inertia of the structure and gravity. This work has been pursued with the apparition of semi-passive walker combining both specific passive properties and low power actuation to increase their robustness [21]. We can note the work of Collins [22] which explored the case of semi-passive 3D biped robot. Its morphology is based on particular mass distribution, knee locking, round feet and springs on the

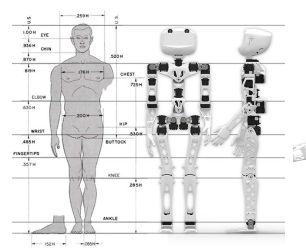

(a)

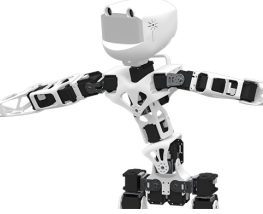

(b)

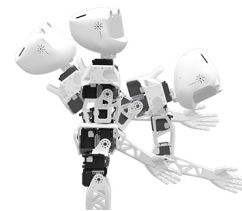

(c)
Fig. 2: These figures illustrate some morphological features of the Poppy humanoid Robot:

a) The Poppy's limbs follow the human being proportion as described in [31].

b) and c) Poppy has an articulated trunk of 5 DoFs which allows more natural and fluid motions while improving the user experience during physical interaction and actively participating to the balance of the robot.

legs to generate an efficient walking gait while keeping its lateral and frontal balance. The concept of 3D semi-passive robot has been pushed even further with the realization of a complete humanoid robot with trunk, arms and head: the robot Denise [23] and Flame presented in [24].

Several studies have also explored the role of the foot and ankle morphology for biped walking on both human [25] [26] and robot [27] [28]. However, to our best knowledge no research has focused on the role of the thigh for biped locomotion. While the HRP-4C [29] and Kenshiro humanoid [30] robot seem to visually have a morphology design close to the thigh shape of Poppy, they did not study, in the associated scientific papers the role of this shape on the dynamic of their humanoid robot.

\section{THE POPPY MORPHOLOGY}

Poppy is a kid-size $(84 \mathrm{~cm})$ humanoid robotic platform designed for scientific experiments on biped locomotion and human-robot interaction. It easily permits the trial of several morphologies and learning algorithms. For this purpose, the morphological optimization is mainly expressed on the locomotive system (legs and trunks) to increase the robot robustness, agility and stability during the walking.

In this section we will first present an overview of the general morphological properties of the robot. Then we will focus on the thigh shape and explain its role with a theoretical model. While the general platform has been described in [9], we focus here on the description of the theoretical model of the thigh shape and we introduce an experimental evaluation of its role in biped locomotion.

\section{A. Morphology adapted for biped walking}

The Poppy robot was conceived following these design goals:

Anatomical proportion are bio-inspired: The Poppy's limbs respect the proportion of the human being (see Fig. 2a).

Large sensorimotor-space: Poppy has a large sensorimotor space composed of 25 Robotis Dynamixe ${ }^{1}$ servomotors

http://www.robotis.com/xe/dynamixel_en 
(23 MX-28 and 2 AX-12). These servo-motors give access to a large number of internal sensors and allow the dynamical tuning of their compliance. The sensors space is extended by the addition of 8 force sensors under each foot, an inertial measurement unit located in the head and two wide-angle HD cameras. The total sensorimotor space is composed by more than 150 dimensions giving access to several kinds of data (e.g. position, speed, load, temperature, acceleration, foot pressure).

Articulated spine: Poppy has 5 motors in the trunk allowing the reproduction of the main DOFs of the human spine [32] (see Fig. 2b and Fig. 2c). These DOFs enable more natural and fluid motions while improving the user experience during physical interaction [33]. In addition, the spine plays a fundamental role in bipedal walking and postural balance by actively participating in the balancing of the robot.

Light-weight: Mechanical parts are 3D printed using Polyamide ${ }^{2}$ material and selective laser sintering technics. They were designed to optimize the weight to make the Poppy robot as light as possible. The obtained mass reduction allows the use of less powerful motor and therefore lighter, resulting in a lightweight $(3.5 \mathrm{~kg})$ and robust robot with sufficient power for a wide range of tasks.

Small feet with compliant toes: To allow efficient and human-like walking gait, Poppy's feet design takes some functional inspiration from the actual human foot such as the proportion (i.e. small compared to conventional humanoids), compliance and toes which are key features concerning both the human walking [26] and biped robots with a human-like gait [34].

Semi-passive knees: Traction springs placed on knees help to keep the legs straight during the support phase without any motor control.

Bio-inspired bended thigh: The shape of the thigh is inspired of the human thigh. It is bended by angle of $6^{\circ}$, increasing the stability of the robot. Its design and effect will be discuss in details in the section III-B and experiments will be conducted to evaluate its effect on the robot dynamic section IV.

\section{B. Effect of the bended thigh}

If we look closely at the human morphology of the femur, it appears that it is inclined of $6^{\circ}$. This makes the feet closer to the projection of the center of gravity (see Fig. 3a. This approach leads to two main stability enhancements during the walking gait:

1) Reduce the falling speed during single support phase: We can model the situation where the robot is on one foot by an inverted pendulum with a point mass centered on the center of gravity $(\mathrm{CoG})$ of the robot and the axis of rotation located at the foot position (see Fig. 3c). The dynamic of the whole structure depends on:

- the length $l$ of the segment extending from the foot to the center of gravity,

2 http://i.materialise.com/materials/polyamide

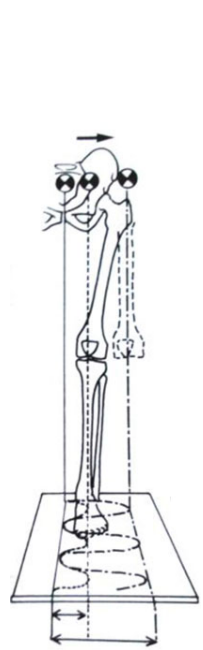

(a)

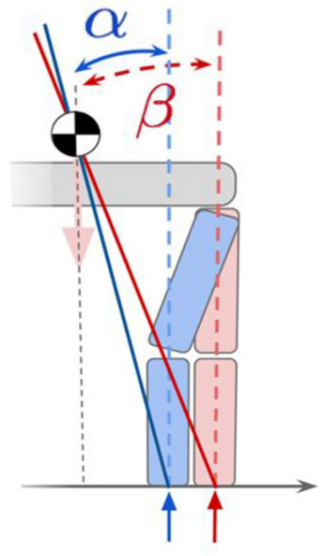

(b)

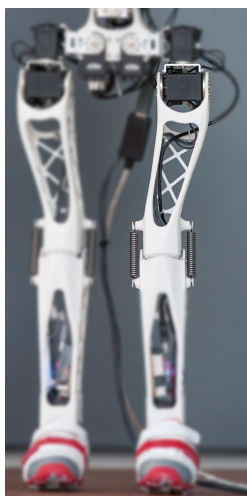

(c)
Fig. 3: a) Effect of the human being bended femur on the human biped locomotion. b) Model used for the comparison of the two thighs morphology. c) Actual realization of the bended thigh on the poppy platform

- the angle $\theta$ of the segment relative to the vertical,

- the gravity force $g$.

The system follows the law:

$$
\begin{gathered}
\ddot{\theta}(t)+w_{0} \cdot \sin (\theta(t))=0 \\
w_{0}=\sqrt{\frac{g}{l}}
\end{gathered}
$$

To get a first idea of the behavior, we can linearize the system for small disturbance such as:

$$
\begin{gathered}
\theta(t)=\theta_{0} \cdot \cos \left(w_{0} \cdot t\right) \\
\dot{\theta}(t)=-\theta_{0} \cdot w_{0} \cdot \sin \left(w_{0} \cdot t\right)
\end{gathered}
$$

The position and velocity of the pendulum linearly varies with the initial $\theta$ angle. Reducing this initial angle $\theta_{0}$ involves a direct reduction of the falling speed $\dot{\theta}(t)$ of the robot.

In the case of Poppy's geometry, the thigh bending allows a $40 \%$ reduction of the initial angle $\theta_{0}\left(\alpha=3.8^{\circ}\right.$ against $\beta=6.4^{\circ}$ on Fig. 3b.

In the case of a fall, it is not possible to respect the assumption of small perturbations, that is why we have simulated the model in Matlab with a non-linear system. We obtain the behavior represented in Fig. 4

If we define the center of gravity altitude as:

$$
z_{C o G}=l \cdot \cos (\theta(t))
$$

We can express its falling speed over time as:

$$
\dot{z}_{C o G}=-\dot{\theta}(t) \cdot l \cdot \sin (\theta(t))
$$

The simulation shows that between 0 and $700 \mathrm{~ms}$, the mean of the CoG falling speed is reduced by $56 \%$. 

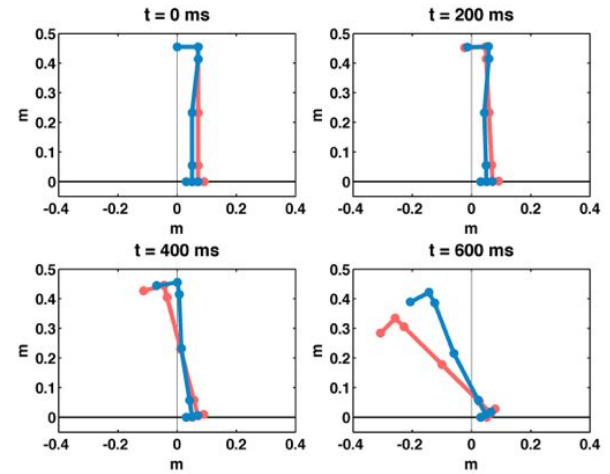

Fig. 4: Comparison of the falling dynamic over time when Poppy is standing on one foot depending on its thigh morphology: with a bended thigh of $6^{\circ}$ (blue) and with a straight thigh (red).

2) Reduce the lateral translation of the center of gravity during double stance phase: As the feet are closer to the gravity center, the necessary lateral translation of the $\mathrm{CoG}$ to transfer the mass of the robot from one foot to another is reduced (see Fig 3a). In the case of Poppy's morphology, thanks to the $6^{\circ}$ bended thigh, the lateral motion of the CoG is reduced by about $30 \%(5 \mathrm{~cm}$ instead of $7.1 \mathrm{~cm})$.

\section{EXPERIMENTS}

The simple model described in the previous section showed that a slight inclination $\left(6^{\circ}\right)$ of the thigh can theoretically have a significant gain regarding the lateral stability of the robot during the two main phases of the walking gait (i.e. single stance phase III-B.1 and double stance phase III-B.2.

In this section, we describe representative experiments which evaluate the actual gain of the thigh shape on the real Poppy platform. To do this, we used both a pair of straight thighs and the bended thighs presented above. We will compare Poppy's reactions with those different legs (see Fig. 11 on three experiments:

A. Evaluate the falling speed during single support stance.

B. Measure the lateral translation to move the CoG Form one feet to the other.

C. Record the upper body motion during biped locomotion.

\section{A. Single support falling velocity}

The experiment evaluates the fall velocity of Poppy when it is supported on only one foot and compare it with the theoretical results obtained in III-B.1 To do so, the robot's head is tracked by an Optitrack ${ }^{3}$ device and markers are placed on the head. In postural balance on two feet, a motor order triggers the raise of a foot which unbalances the robot (see Fig. 5a) and causes its lateral fall (see Fig. 5b). This experiment was repeated about fifteen times for the two cases studied, i.e. with bended legs (Fig. 1a) and with straight legs (Fig. 1b.

3 http://www.naturalpoint.com/optitrack/products/ v120-trio/

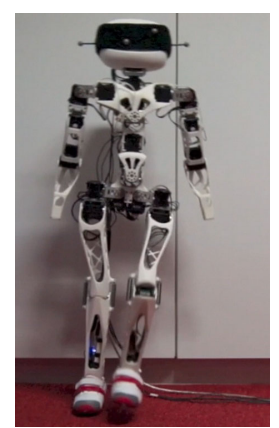

(a)

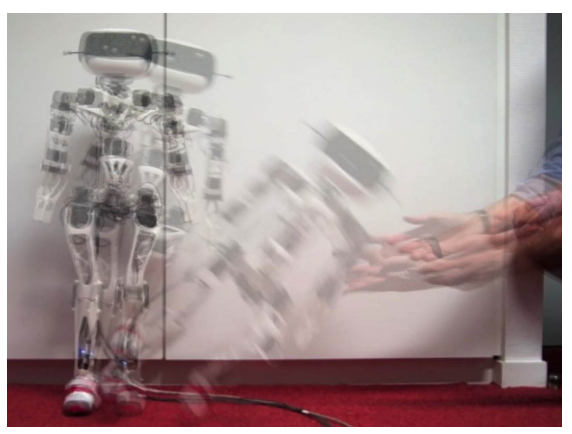

(b)
Fig. 5: Proceedings of the one support stance falling experiment. The Poppy head has a headband with markers to track its absolute position over time. a) Initial perturbation done a sudden raise of one foot, b) view of the Poppy lateral fall over time.

Experiments results are shown on the Fig. 6 The blue color is assigned to experiments with bended thighs while the red color is assigned to straight thighs. For each case, the light color corresponds to the standard deviation and the dark color to the $95 \%$ confidence interval of the mean value. The first figure 6a refers to the head altitude position over time and the second $6 \mathrm{~b}$ ) to the falling velocity of the head. Dashed lines represent theoretical results obtained with the model presented in section $3 \mathrm{~b}$. One can notice the strong similarity both on the shape and on the difference between the two morphologies studied. Yet, there is a slight time shift between theoretical and experimental results. This can be explained by the inertia of the real robot which was not take into account during the simulation.

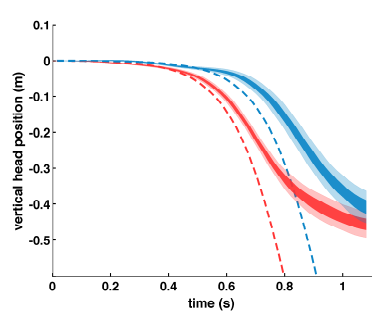

(a) Vertical head position

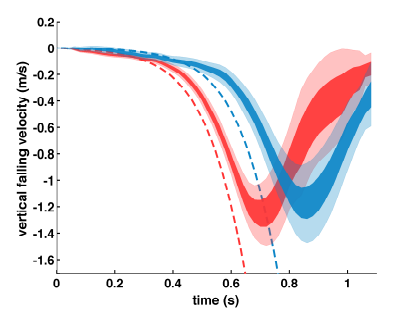

(b) Vertical head falling velocity
Fig. 6: Results of the single support falling experiment. The blue color is associated with experiments conducted with bended thighs while the red color is assigned to straight thighs. For each case, the light color corresponds to the standard deviation and the dark color to the $95 \%$ confidence interval of the mean value while dashed lines represent theoretical results. These figures shows the vertical position (a) and vertical falling velocity (b) of the head of Poppy over time for each case studied. The curves behavior change after $800 \mathrm{~ms}$ is due to the fact that we catch up the robot before it touches the ground.

These figures show a clear improvement for the Poppy 
version with bended thigh (blue curves) with a $200 \mathrm{~ms}$ time shift compared to the straight thigh (as illustrated on the attached video ${ }^{4}$. Thanks to this delay, the falling speed is reduced by about $56 \%$ during the first $700 \mathrm{~ms}$. Thus the robot remains almost stationary for $600 \mathrm{~ms}(400 \mathrm{~ms}$ in the case of straight thigh). The typical walking gait of Poppy is done over a period of one second so the mono-pedal stance phase last around $420 \mathrm{~ms}$ [9]. Considering that the robot remains stationary during more time than the single stance phase, we can imagine that the lateral balance control will be reduced during the walking gait.

\section{B. Double support CoG transfer}

In this experiment we evaluate the necessary lateral movement of the robot to cause a displacement of its center of gravity from one foot to the other and verify the theoretical results obtained in III-B.2 For this, Poppy is placed on a force platform to measure the displacement of its center of pressure. The absolute movements of the robot are tracked with an OptiTrack device and markers placed at the head and lower back (approximately the position of the actual center of gravity). The robot is kept rigid in a neutral position and a human physically pushed it from left to right until it reaches its lateral falling limit. As this operation is not very accurate, the experiment is repeated a hundred times.

\begin{tabular}{|l|c|c|c|}
\hline & Straight tigh & Bended Tigh & diff(\%) \\
\hline CoP & $74.6 \pm 9.0 \mathrm{~mm}$ & $49.8 \pm 7.7 \mathrm{~mm}$ & 33 \\
Head & $100.1 \pm 14.4 \mathrm{~mm}$ & $62.9 \pm 22.0 \mathrm{~mm}$ & 37 \\
Lower Back & $64.1 \pm 11.5 \mathrm{~mm}$ & $43.4 \pm 15.0 \mathrm{~mm}$ & 32 \\
\hline
\end{tabular}

TABLE I: Summary of the results obtained during the experiment on the lateral motion needed to transfer the robot mass from one foot to the other.

The table $\mathrm{I}$ presents for each area considered (i.e. center of pressure (under feet), lower back and head motion) the amplitude of the lateral motion (in millimeter) needed to translate the $\mathrm{CoG}$ of the robot from one foot to the other for the two versions of the Poppy thigh design. The last columns summarizes the relative difference between the two conceptions (in percent). One can note that the results show a reduction of lateral movement of around $30 \%$. Thanks to the shape of the thigh, the lateral displacement of the upper body required to move the $\mathrm{CoG}$ from one foot to the other can be reduced.

The results presented on the two first experiments show improvement for two main aspects needed during biped locomotion: lateral stability and mass transfer. In the next experiment, we will evaluate if there is a significant performance gain in a complex dynamic phase such as bipedal walking.

\section{Walking dynamic}

As explained in the introduction and description of the platform, Poppy has been especially designed to study bipedal walking and human-robot interaction.

http://flowers.inria.fr/Humanoid2013/
Here the experiment consists in playing an open-loop walking pattern while the robot is guided through the physical interaction with a human. The users role is to provide both balance and control of mass transfer. By producing small lateral motion on the upper-body they can help the robot to move its $\mathrm{CoG}$ from one foot to another. (a)

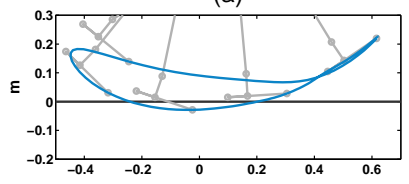

(c)

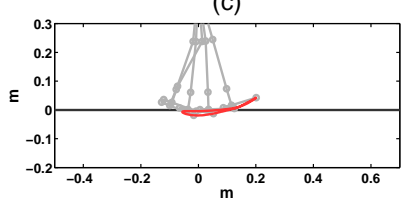

(b)

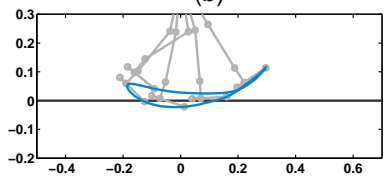

(d)

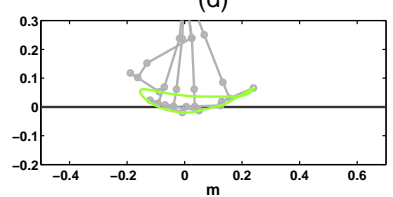

Fig. 7: Toes trajectories generated by the walking pattern a) Kinematics of human walking with human's morphology b) Direct transposition of the human kinematics with Poppy's morphology c) Reducing amplitude of the human kinematics joints with Poppy's morphology d) Walking pattern used for the experiment with Poppy

The gait is based on the actual human sagittal joint kinematic [35]: hip, knee, ankle (see Fig. 7.a). A direct transposition of the human joint kinematic on the Poppy's morphology results in a walking speed which is too fast to be handled by users (see Fig. 7b). A simple reduction of joints amplitude conducts to an unsuitable leg trajectory where toes bump into the ground during the swing phase (see Fig. 7.c). So to ensure enough clearance during the swing phase and suitable walking speed for the guidance with user, we modified the joints trajectories by hand to both reduce the length step and increase the foot clearance (see Fig. 7 d). The actual gait on Poppy is shown on the Fig. 8 .

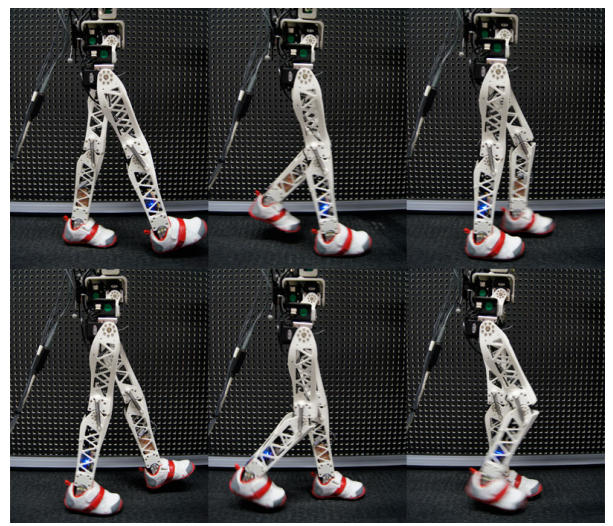

Fig. 8: Walking gait CPG described on Fig. 7.d applied on the actual Poppy robot. The CPG generates a human-like walking gait allowing the robot to walk at $1.8 \mathrm{~km} / \mathrm{h}$ and involves straight leg during the stance phase. There is no balance control but stability is obtained through physical guidance with trained expert user. 
In this experiment we are interested by the dynamic of Poppy especially on the frontal plane and we will compare the effect of the thigh shape on this dynamic. Poppy walks on a treadmill following the walking gait described above. An expert user trained to keep the robot in the correct walking cycle provides guidance to the robot. This is done by keeping the robot in a vertical position and supporting, in a compliant manner, the lateral movement of the robot as illustrated in attached videos ${ }^{4}$. The user is asked to do the best he can to minimize the movement/forces he applies in both morphologies to reduce the bias towards the two design experimented. All proprioceptive sensors are recorded at $50 \mathrm{hz}$ while an Optitrack device associated to markers located at the head and lower back measure the absolute displacements of the robot (voir Fig. 9).

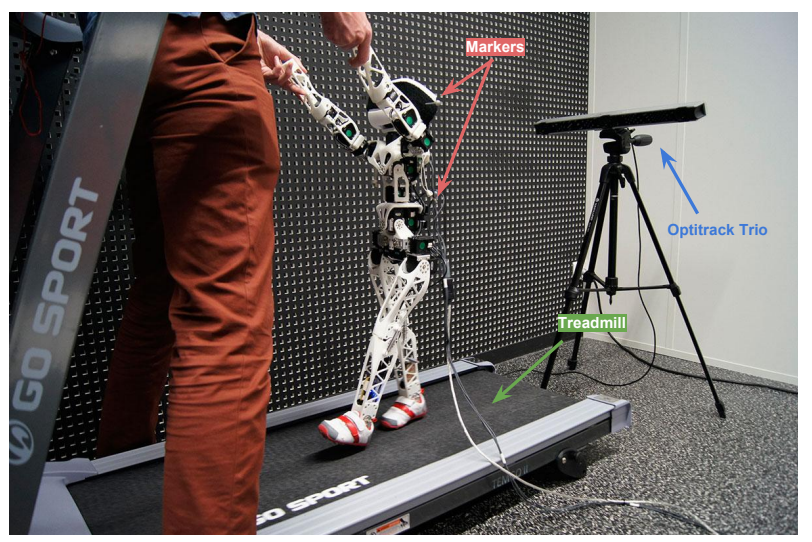

Fig. 9: Proceeding of the walking experiment. Poppy is tracked by an Optitrack trio while it is walking on a treadmill set at $1.8 \mathrm{~km} / \mathrm{h}$. An expert user provides the sagittal balance needed during all the experiment.

The Poppy dynamic is recorded for around 1800 walking gait cycle for each thigh design (around 90,000 data points for each case). Data are folded over to extract the gait behavior over a gait cycle. Results are presented on Fig. 10 . As previously, the blue color is assigned to experiments with bended thigh, the red color is assigned to straight thigh. For each case, the light color corresponds to the standard deviation and the dark color to the $95 \%$ confidence interval of the mean value.

The two first figures (i.e. 10a and 10b) show the upper body lateral motion in millimeter over the gait cycle. We can notice that for the two designs, the motion pattern shown by the upper body (head and lower back) is similar. However in the case of the bended thigh (blue) the amplitude of the motion is reduced by about $45 \%$. Another interesting effect concerns the head perturbations shown on figures $10 \mathrm{c}$, and 10d Here also, patterns are similar but in the case of the bended thigh the head is clearly less perturbed by the walking dynamic with a reduction in amplitude of approximately $30 \%$.

Five pictures have been taken while Poppy was walking and were stacked on Fig. 11 This shows a qualitative point of view of the walking dynamic for both studied case. We

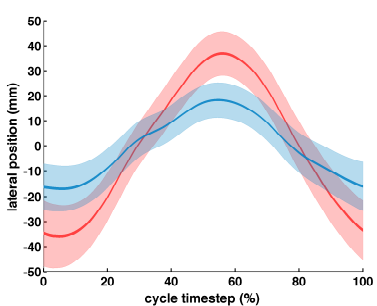

(a) Lateral head displacement

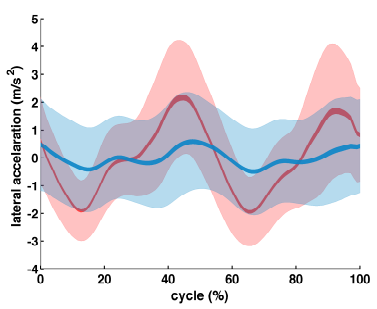

(c) Sagittal head acceleration

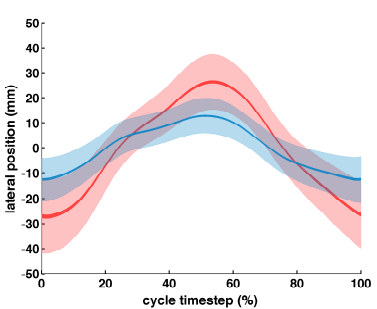

(b) Lateral lower back displacement

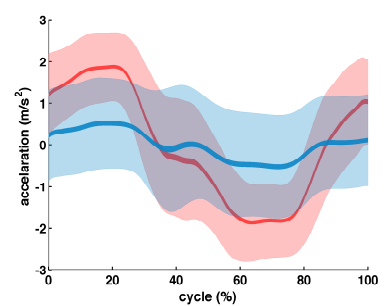

(d) Lateral head acceleration
Fig. 10: Results obtained during the walking experiment. The blue color is associated with experiments conducted with bended thighs while the red color is assigned to straight thighs. For each case, the light color corresponds to the standard deviation and the dark color to the $95 \%$ confidence interval of the mean value. All data are folded over to extract the mean gait behavior and its standard deviation over a walking gait cycle expressed in percent.

can notice that the lateral motion of the version of Poppy with bended thigh 11a is small compared to the version with straight thigh $11 \mathrm{~b}$

\section{DisCUSSION \& CONCLUSION}

We have presented the morphological properties of a new humanoid robot platform named Poppy [9]. In this paper, we focus on the shape of the Poppy thigh and its effect on the robot dynamic. We studied the role of the morphology in the reduction and simplification of the control needed to performs complex task such as biped walking. We have presented the simple theoretical model we used for the design of Poppy thigh based on the inverse pendulum dynamic. We have conducted experiments to evaluate the improvements of the bended thigh on the real robot dynamic and compare it with the model. Thanks to the conception of Poppy allowing easy, cheap and fast morphology modifications, we were able to try another thigh design. We also use a pair of straight thighs which is a more classical approach in humanoid conception. The experimental comparison of the two thighs design confirmed the theoretical results, the bio-inspired thigh design improves Poppy dynamic on two main points useful for biped walking:

- It reduces the falling velocity by almost $60 \%$ when the robot is on one foot (single support phase).

- It reduces by $30 \%$ the lateral motion needed to transfer the mass of the robot from one foot to the other (double support phase). 


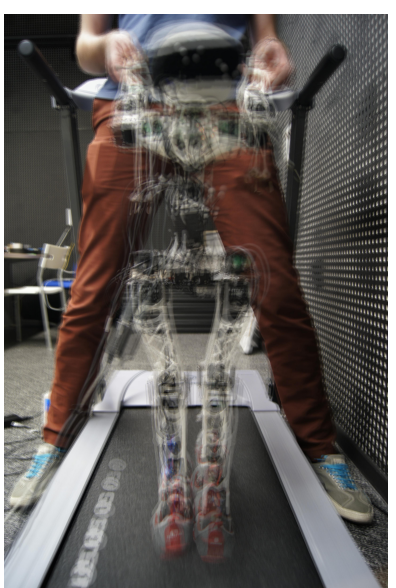

(a) bended thigh

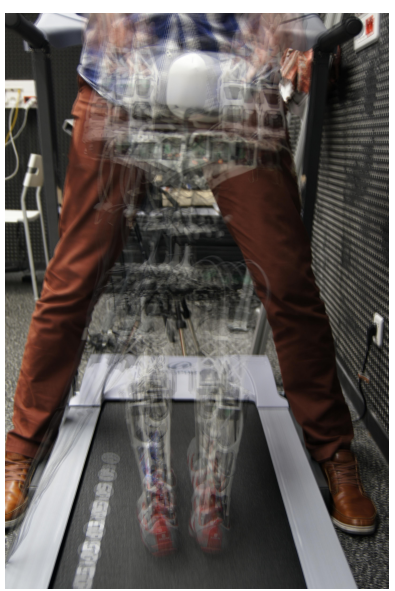

(b) straight thigh

Fig. 11: Five pictures have been taken while Poppy was walking and were stacked to obtain a qualitative view of the difference in the walking behavior in function of the morphology of the thigh.

It is really interesting to note that such a small modification of the robot morphology has a very significant impact on the robot behavior.

These results are interesting but they do not reflect the actual Poppy dynamic while it is walking. To evaluate the effect of the bended thigh on the biped locomotion, we conducted a third experiment where Poppy is walking on a treadmill. In this experiment, we show that the bended thigh has an effect on a complex dynamic task such as the biped locomotion: it reduces the motion amplitude on the upper body of $45 \%$ and increase the head stability of $30 \%$. We choose these metrics due to our experimental constraints (fixed speed, social guidance) as a qualitative evaluation of the walking gait. Moreover it provides us with an intuitive, yet incomplete evaluation of the walking. Many other measures could have been chosen or combined such as speed, energy consumption or robustness to external perturbations. It is still complicated to understand which metric is the most adapted for the robotic biped locomotion. As human being is trained to recognize biped gait, users can provide guidance to the robot for both safety of exploration and evaluation of the walking behavior.

We will thus, in our future work, study the role of morphology in the learning of biped locomotion through this interaction. Thanks to its multi-articulated and compliant spine (see Fig. 2), users physically induce motion in the trunk motors while they are guiding Poppy. We could imagine learning this motion needed to generate the mass transfer during the walking gait. Also, as the upper motions of Poppy with the bended thigh are small, we can imagine that the learning will be simplified. An other interesting aspect concerns the evaluation of the right walking metrics to optimize. As the guidance given by users is safe and provides good demonstrations of a "correct" walking gait, the robot can record a large amount of useful data to create a representation of the right walking behavior and find an adapted metric to evaluate its learning performances.

Last but not least, Poppy was conceived while considering issues of dissemination and was optimized to be very accessible (i.e. cheap and easy to assemble). Thus the whole Poppy drawing and software will be shortly released under OpenSource license for academics. They will be able to reproduce the results obtained in this article, investigate other morphologies or use it as a test-bench for learning algorithms and human-robot interaction.

\section{REFERENCES}

[1] Kazuo Hirai, Masato Hirose, Yuji Haikawa, and Toru Takenaka. The development of Honda humanoid robot. In Robotics and Automation, 1998. Proceedings. 1998 IEEE International Conference on, volume 2, pages 1321-1326. IEEE, 1998.

[2] Kenji Kaneko, Kensuke Harada, Fumio Kanehiro, Gou Miyamori, and Kazuhiko Akachi. Humanoid robot HRP-3. In Intelligent Robots and Systems, 2008. IROS 2008. IEEE/RSJ International Conference on, pages 2471-2478. IEEE, 2008.

[3] Shuuji Kajita, Fumio Kanehiro, Kenji Kaneko, Kiyoshi Fujiwara, Kensuke Harada, Kazuhito Yokoi, and Hirohisa Hirukawa. Biped walking pattern generation by using preview control of zero-moment point. In Robotics and Automation, 2003. Proceedings. ICRA'O3. IEEE International Conference on, volume 2, pages 1620-1626. IEEE, 2003.

[4] A Billard, S Calinon, R Dillmann, and S Schaal. Robot programming by demonstration. Handbook of robotics, 1, 2008.

[5] Adrien Baranes and Pierre-yves Oudeyer. Active learning of inverse models with intrinsically motivated goal exploration in robots. Robotics and Autonomous Systems, 2012.

[6] Rolf Pfeifer and Fumiya Iida. Morphological computation: Connecting body, brain and environment. Japanese Scientific Monthly, 58(2):4854, 2005.

[7] Rolf Pfeifer and Josh C Bongard. How the Body Shapes the Way We Think: A New View of Intelligence (Bradford Books). The MIT Press, 2006.

[8] Rolf Pfeifer, Max Lungarella, and Fumiya Iida. Self-organization, embodiment, and biologically inspired robotics. Science, 318(5853):1088-1093, November 2007.

[9] Matthieu Lapeyre, Pierre Rouanet, and Pierre-Yves Oudeyer. The Poppy Humanoid Robot: Leg Design for Biped Locomotion. In IEEE/RSJ International Conference on Intelligent Robots and Systems, Tokyo, Japon, 2013.

[10] Jong H Park and Kyoung D Kim. Biped robot walking using gravitycompensated inverted pendulum mode and computed torque control. In Robotics and Automation, 1998. Proceedings. 1998 IEEE International Conference on, volume 4, pages 3528-3533. IEEE, 1998.

[11] Shinya Aoi and Kazuo Tsuchiya. Locomotion control of a biped robot using nonlinear oscillators. Autonomous Robots, 19(3):219-232, 2005.

[12] Miomir Vukobratović and Branislav Borovac. Zero-moment point-thirty five years of its life. International Journal of Humanoid Robotics, 1(01):157-173, 2004.

[13] Kazuhiko Akachi, Kenji Kaneko, Noriyuki Kanehira, Shigehiko Ota, G. Go Miyamori, Masaru Hirata, Shuuji Kajita, and Fumio Kanehiro. Development of humanoid robot HRP-3P. In Humanoid Robots, 2005 5th IEEE-RAS International Conference on, pages 50-55. IEEE, Ieee, 2005.

[14] Uluc Saranli, Martin Buehler, and Daniel E Koditschek. Rhex: A simple and highly mobile hexapod robot. The International Journal of Robotics Research, 20(7):616-631, 2001.

[15] Chandana Paul and Josh C Bongard. The road less travelled: Morphology in the optimization of biped robot locomotion. In Intelligent Robots and Systems, 2001. Proceedings. 2001 IEEE/RSJ International Conference on, volume 1, pages 226-232. IEEE, Ieee, 2001.

[16] Ken Endo, Takashi Maeno, and Hiroaki Kitano. Co-evolution of morphology and walking pattern of biped humanoid robot using evolutionary computation. Consideration of characteristic of the servomotors. In Intelligent Robots and Systems, 2002. IEEE/RSJ International Conference on, volume 3, pages 2678-2683. IEEE, 2002. 
[17] Hartmut Geyer, Andre Seyfarth, and Reinhard Blickhan. Compliant leg behaviour explains basic dynamics of walking and running. Proceedings. Biological sciences / The Royal Society, 273(1603):2861-7, November 2006.

[18] Kojiro Matsushita, Max Lungarella, Chandana Paul, and Hiroshi Yokoi. Locomoting with less computation but more morphology. In Robotics and Automation, 2005. ICRA 2005. Proceedings of the 2005 IEEE International Conference on, pages 2008-2013. IEEE, Ieee, 2005.

[19] Martijn Wisse, Guillaume Feliksdal, J Van Frankkenhuyzen, and Brian Moyer. Passive-based walking robot. Robotics \& Automation Magazine, IEEE, 14(2):52-62, 2007.

[20] Tad McGeer. Passive dynamic walking. The International Journal of Robotics Research, 9(2):62-82, 1990.

[21] S.O. Anderson, M. Wisse, C.G. Atkeson, J.K. Hodgins, G.J. Zeglin, and B. Moyer. Powered bipeds based on passive dynamic principles. 5th IEEE-RAS International Conference on Humanoid Robots, 2005. pages 110-116, 2005.

[22] SH Steven Hartley Collins and Andy Ruina. A bipedal walking robot with efficient and human-like gait. In Robotics and Automation, 2005. ICRA 2005. Proceedings of the 2005 IEEE International Conference on, pages 1983-1988. IEEE, 2005.

[23] Martijn Wisse. Three additions to passive dynamic walking: actuation, an upper body, and 3D stability. International Journal of Humanoid Robotics, 2(04):459-478, 2005.

[24] D. Hobbelen, T. de Boer, and M. Wisse. System overview of bipedal robots Flame and TUlip: Tailor-made for Limit Cycle Walking. 2008 IEEE/RSJ International Conference on Intelligent Robots and Systems, pages 2486-2491, September 2008.

[25] Peter G Adamczyk, Steven H Collins, and Arthur D Kuo. The advantages of a rolling foot in human walking. The Journal of experimental biology, 209(Pt 20):3953-63, October 2006.

[26] J Hughes, P Clark, and L Klenerman. The importance of the toes in walking. The Journal of bone and joint surgery. British volume, 72(2):245-51, March 1990

[27] Daan G E DGE Hobbelen and Martijn Wisse. Ankle joints and flat feet in dynamic walking. In Climbing and Walking Robots, pages 787-800. Springer, 2005.

[28] S Davis and D G Caldwell. The design of an anthropomorphic dexterous humanoid foot. 2010 IEEE/RSJ International Conference on Intelligent Robots and Systems, pages 2200-2205, October 2010.

[29] Kenji Kaneko, Fumio Kanehiro, Mitsuharu Morisawa, Kanako Miura, Shin'ichiro Nakaoka, and Shuuji Kajita. Cybernetic human HRP4C. In Humanoid Robots, 2009. Humanoids 2009. 9th IEEE-RAS International Conference on, pages 7-14. IEEE, 2009.

[30] Yuto Nakanishi, Shigeki Ohta, Takuma Shirai, Yuki Asano, Toyotaka Kozuki, Yuriko Kakehashi, Hironori Mizoguchi, Tomoko Kurotobi, Yotaro Motegi, Kazuhiro Sasabuchi, Junichi Urata, Kei Okada, Ikuo Mizuuchi, Masayuki Inab, and Others. Design Approach of Biologically-Inspired Musculoskeletal Humanoids. International Journal of Advanced Robotic Systems, 10(216):1, 2013.

[31] Michel Dufour and M Pillu. Biomécanique fonctionnelle: MembresTête-Tronc. Paris: Masson, 2005.

[32] J C Ceccato. Le tronc, de la locomotion à la commande. $\mathrm{PhD}$ thesis, Montpellier II, 2009.

[33] Olivier Ly, Matthieu Lapeyre, and Pierre-yves Oudeyer. The Humanoid Robot Acroban: Leveraging Semi-Passive Dynamics in the Vertebral Column. 4(1):1-36, 2010.

[34] Ramzi Sellaouti, Olivier Stasse, Shuuji Kajita, Kazuhito Yokoi, and Abderrahmane Kheddar. Faster and Smoother Walking of Humanoid HRP-2 with Passive Toe Joints. 2006 IEEE/RSJ International Conference on Intelligent Robots and Systems, pages 4909-4914, October 2006

[35] C J Nester, M L van der Linden, and P Bowker. Effect of foot orthoses on the kinematics and kinetics of normal walking gait. Gait \& posture, 17(2):180-7, April 2003. 